\title{
Evaluación del sistema de limpieza de una máquina de papel Kraft
}

\author{
Maria J. Tovar*, Lady Sandoval*, Oscar Sabogal**, Edgar Mora**, Fiderman Machuca*§ \\ * Escuela de Ingeniería Química, Facultad de Ingeniería, Universidad del Valle, Cali, Colombia \\ ** SmurfitKappa Cartón de Colombia S.A. \\ §e-mail:fiderman@univalle.edu.co
}

(Recibido: Abril 6 de 2006 - Aceptado: Octubre 27 de 2006)

\begin{abstract}
Resumen
El artículo presenta la metodología y resultados de la evaluación del sistema de limpieza de una máquina de papeles Kraft. Esta evaluación se realizó teniendo en cuenta variables de proceso tales como la consistencia de la suspensión de pulpa, contenido de inorgánicos (cenizas), nivel de aire en la suspensión de pulpa y el diferencial de presión a través de los hidrociclones, para el análisis de una serie de muestreos realizados durante la producción de los diferentes grados de papel. Se propusieron las posibles modificaciones o mejoras del sistema de limpieza con el objetivo de disminuir las pérdidas de pulpa y agua, el consumo de energía y la introducción de aire al sistema en la máquina de papel.
\end{abstract}

Palabras clave: Fábrica de papel, Papeles Kraft, Hidrociclones, Consistencia de pulpa, Contenido de cenizas.

\section{Evaluation of the cleaning system of a Kraft paper mill}

\begin{abstract}
This paper shows the methodology and results of the evaluation of the cleaning system of a Kraft paper mill. This evaluation was made keeping in mind variables of that system such as the pulp stock consistency percentage, ash content, air level in the pulp stock, and differential of pressure through the cleaners, for the analysis of a series of samplings carried out during the production of the different paper grades. On the basis of the results, possible modifications or improvements of the cleaning system were proposed, which will allow a reduction in pulp and water losses, energy consumption, and the amount of air required in the paper machine.
\end{abstract}

Keywords: Paper mill, Kraft papers, Cleaners, Pulp consistency, Ash content. 


\section{Introducción}

El sistema de tamizado y limpieza instalado en una máquina de papel tiene como función la eliminación de los contaminantes presentes en la suspensión de fibras, tales como los shives (paquetes de haces de fibras no cocinadas), arenas, minerales y la fracción reciclada proveniente de papeles Kraft, OCC (Old Corrugated Container) y DKL (Dark Kraft Liner).

Los limpiadores centrífugos que se evaluarán en este trabajo, corresponden a hidrociclones diseñados para eliminar contaminantes con una gravedad específica superior a la de la fibra. Este sistema de limpieza se ha convertido en un punto crítico dentro del proceso, ya que su mal funcionamiento causa pérdidas de producción y mala calidad del papel. En el presente trabajo se realizó un diagnóstico, el cual permitirá tomar decisiones sobre el sistema de limpieza del circuito de aproximación de una máquina de papeles Kraft.

\section{Antecedentes}

El hidrociclón clásico, inventado en 1891, fue usado primero para extraer partículas contaminantes de alta gravedad específica del empaste de fabricación de papel. Sin embargo, su uso no se generalizó en la industria del papel hasta los años 50. En los inicios de los años 70 los hidrociclones clásicos fueron modificados ligeramente permitiendo su uso para la eliminación de contaminantes de baja gravedad específica (Attwood \& Baum, 1998).

La limpieza centrífuga se ha considerado como una alternativa eficiente para la separación de los contaminantes presentes en la pulpa por acción de la fuerza centrífuga, pero es necesario tener en cuenta la rotación de la pulpa dentro del equipo de limpieza seguida de la clasificación de partículas de acuerdo con sus densidades y propiedades hidrodinámicas (Merriman, 1993).

El hidrociclón es un limpiador de pared fija en el cual la acción centrífuga se alcanza por la introducción tangencial de la corriente de alimentación en el equipo. La Figura 1 muestra su funcionamiento. Inicialmente, el flujo de alimentación forma parte del vórtice exterior y se extiende hacia abajo de la cámara de alimentación en la sección del cono, en donde el cuerpo del limpiador empieza a estrecharse. Esto restringe el volumen del limpiador, haciendo que el fluido se salga del vórtice exterior, alejándose de la pared y entrando al vórtice interior en el centro del limpiador (Merriman, 1993).

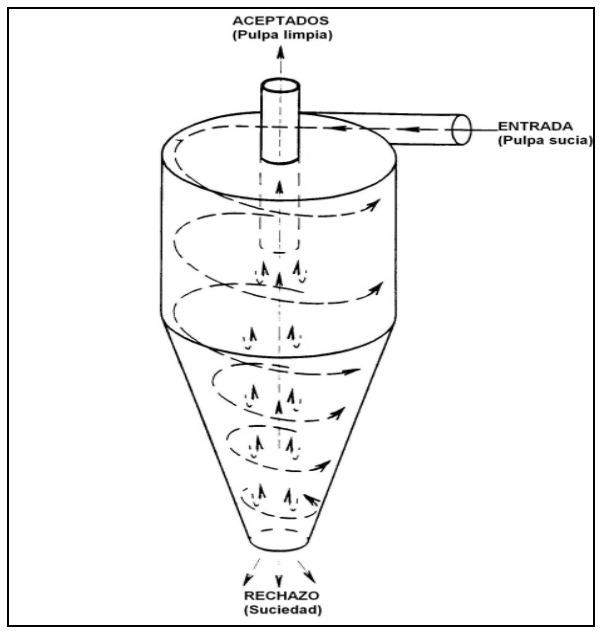

Figura 1. Hidrociclón.

El vórtice interior no solo rota rápidamente sino que a su vez se mueve hacia arriba hasta el orificio de aceptados y hacia abajo a través de la sección cónica hasta el orificio de los rechazos. Al final de la sección cónica, el flujo del vórtice exterior es retirado por el orificio de rechazos.

Las Tablas 1 y 2 presentan un resumen de las variables que afectan al sistema de limpieza y su eficiencia, así como los contaminantes más frecuentes en las fibras recicladas.

Tabla 1. Variables que afectan el funcionamiento de los hidrociclones

\begin{tabular}{|c|c|}
\hline Variables & Variables específicas \\
\hline $\begin{array}{c}\text { Características } \\
\text { de la pulpa }\end{array}$ & $\begin{array}{l}\text { Tipo de fibra } \\
\text { Características de contaminantes (tamaño, forma, densidad) } \\
\text { Nivel de impurezas }\end{array}$ \\
\hline $\begin{array}{l}\text { Diseño del } \\
\text { hidrociclón }\end{array}$ & $\begin{array}{l}\text { Diámetro del cuerpo } \\
\text { Configuración de la entrada de alimentación } \\
\text { Diámetro de la salida de aceptados } \\
\text { Altura de la sección cilíndrica } \\
\text { Ángulo del cono } \\
\text { Existencia de ranuras } \\
\text { Método de control de caudal de rechazos (orificio fijo y contrapresión) }\end{array}$ \\
\hline $\begin{array}{c}\text { Variables } \\
\text { operacionales }\end{array}$ & $\begin{array}{l}\text { Caudal de la pulpa } \\
\text { Caída de presión en el hidrociclón } \\
\text { Relación de rechazos } \\
\text { Temperatura de la pulpa } \\
\text { Disposición de la cámara de descarga }\end{array}$ \\
\hline
\end{tabular}


Tabla 2. Contaminantes de fibras recicladas (Merriman, 1993)

\begin{tabular}{lcl}
\hline \multicolumn{1}{c}{ Tipo } & Gravedad Especifica & $\begin{array}{c}\text { Tamaño de } \\
\text { Particula* }\end{array}$ \\
\hline Arena & $20-22$ & $2,3,4$ \\
Metales & $6.0-9.0$ & 5 \\
Caolín & $1.8-2.6$ & 2 \\
Cera & $0.9-1.0$ & 1,2 \\
Polietileno & $0.91-0.97$ & 4,5 \\
Poliestireno & $1.04-1.10$ & 4,5 \\
Látex & $0.9-1.1$ & 3,4 \\
Asfalto & $1.1-1.5$ & 2,3 \\
Tinta & $1.2-1.6$ & $1,2,3$ \\
Styrofoam & $0.3-0.5$ & 4,5 \\
Fundidos & $0.95-1.1$ & 3,4 \\
\hline
\end{tabular}

* Tamaños:

1. Parcialmente disuelto

2. Menos de 40 micras

3. 40 - 400 micras

4. $400-4000$ micras

5. Mayor a 4000 micras

\subsection{Características del proceso}

\subsubsection{Sistema de limpieza}

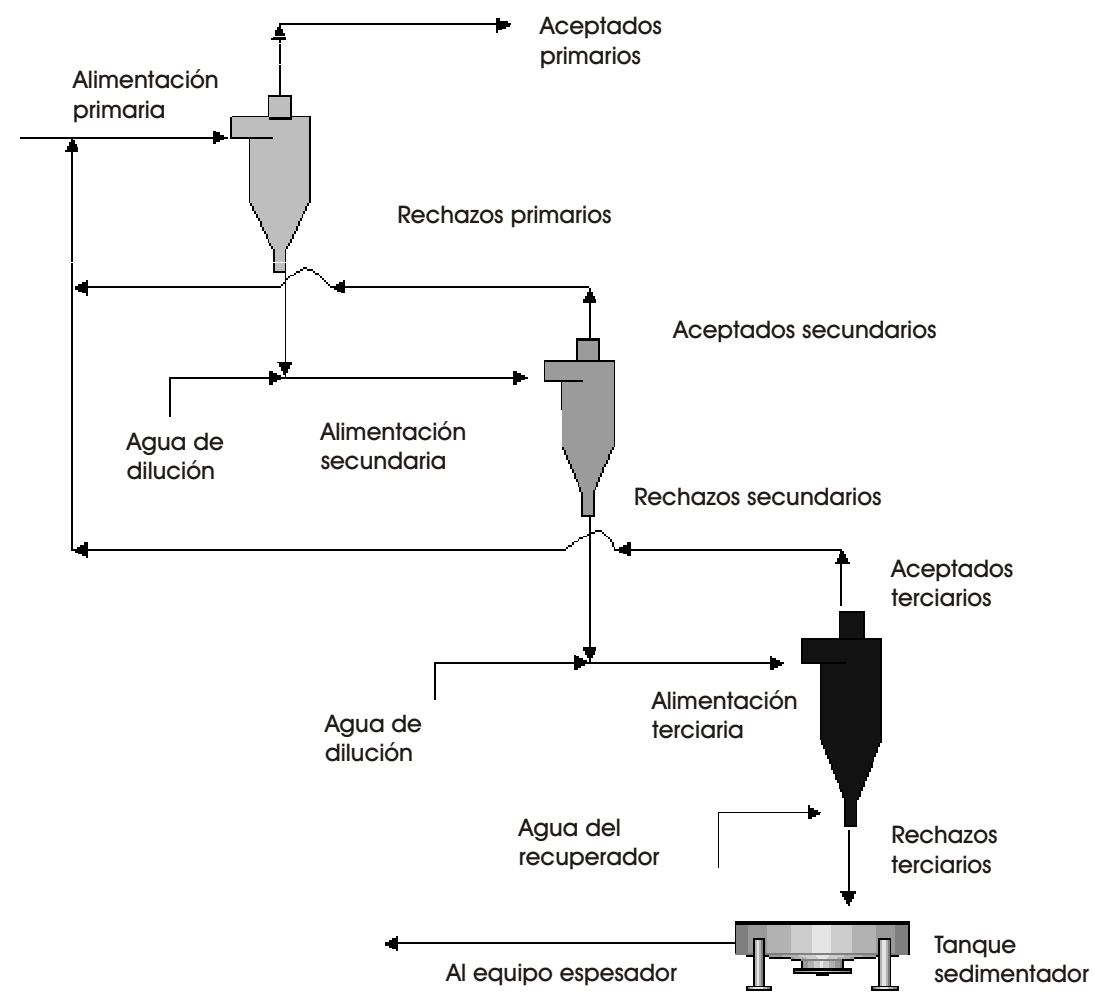

Figura 2. Sistema de limpieza.
La Figura 2 presenta el esquema del sistema de limpieza en estudio, correspondiente al circuito de aproximación de una máquina de papel Kraft, el cual consta de una serie de hidrociclones dispuestos en un sistema multifase (etapa primaria, etapa secundaria y terciaria), lo que permite mayor eliminación de partículas contaminantes. La distribución de los hidrociclones corresponde a un gran número de unidades individuales en una disposición vertical abierta, con líneas de alimentación y de aceptados, con una descarga abierta a la bandeja de rechazos de cada etapa. La proporción de rechazos para la etapa primaria, secundaria y terciaria es de $15-18 \%, 20-25 \%$ y $0.5 \%$, respectivamente.

La etapa primaria consta de 92 hidrociclones, la etapa secundaria de 23 hidrociclones y la etapa terciaria de 7 hidrociclones.

Los aceptados de la primera etapa son diluidos en la succión de la bomba de alimentación para ingresar al proceso de formación del papel, con una consistencia de $0.2 \%-0.7 \%$ dependiendo del 
grado de papel producido y sus rechazos son procesados en cascada a través de la etapa secundaria con una consistencia promedio de $0.9 \%$. Los aceptados de la segunda etapa se unen a la alimentación de la primera etapa y los rechazos son enviados a la tercera etapa. En la tercera etapa los aceptados ingresan a la corriente de alimentación de la primera etapa y los rechazos son llevados a un tanque sedimentador. De allí son bombeados al equipo espesador (hydrasieve) cayendo finalmente al tanque de desperdicio.

\subsubsection{Eficiencia de eliminación}

El comportamiento del sistema de hidrociclones se mide normalmente, en términos de su capacidad para eliminar las partículas de suciedad (Smith, 1979), la cual se calcula con la Ec. (1):

$$
E(\%)=\left(\frac{\% r_{a}-\% \text { rechazos }}{\% r_{a}}\right) * 100
$$

donde $\mathrm{E}(\%)$ es el porcentaje de eficiencia, $\% \mathrm{r}_{\mathrm{a}}$ es el porcentaje de rechazos que entra a la sección y $\% \mathrm{r}_{\mathrm{ac}}$ es el porcentaje de aceptados de la sección.

Los porcentajes de rechazos de alimentación y aceptados se muestran posteriormente en la Tabla 4. La cantidad de pulpa rechazada influye enormemente en la eficiencia de los hidrociclones. Normalmente la relación de rechazos se encuentra en el rango de 10 al $20 \%$.

Tabla 3. Consistencias y cenizas promedio en las entradas a la máquina y al espesador.

\begin{tabular}{cccr}
\hline & Supersacks & $\begin{array}{c}\text { Corrugado } \\
\text { medio }\end{array}$ & Liner \\
\cline { 1 - 2 } Pulpa Pino & & & \\
\% Consistencia & 2.62 & 1.84 & 2.31 \\
\% Cenizas & 0.67 & 0.72 & 0.68 \\
\hline Pulpa Semiquímica & & & \\
\% Consistencia & & 3.77 & 5.03 \\
\% Cenizas & & 1.20 & 1.07 \\
\hline Hydrasieve & & & \\
\% Consistencia & 0.87 & 0.90 & 0.99 \\
\% Cenizas & 1.11 & 2.18 & 1.66 \\
\hline Pulpa Semiquímica Patio & & & \\
\% Consistencia & & 26.95 & 25.45 \\
\% Cenizas & & 1.46 & 1.37 \\
\hline
\end{tabular}

La eficiencia de los hidrociclones, es afectada por la consistencia de la alimentación, el diferencial de presión entre la alimentación y los aceptados, la relación de rechazos y las condiciones físicas de los mismos.

\section{Metodología}

La Figura 3 presenta la metodología utilizada para el análisis del sistema de limpieza y los lugares de muestreo en el sistema.

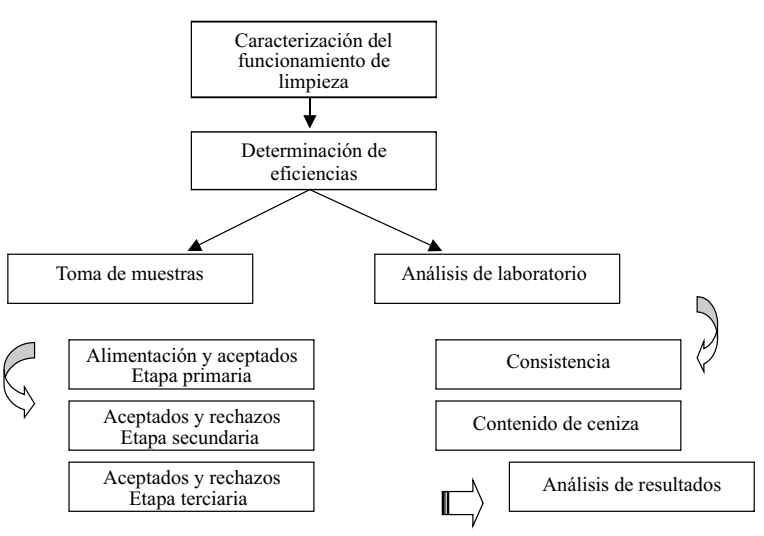

Figura 3. Metodología de evaluación.

A las muestras se les realizaron pruebas de laboratorio para determinar su contenido de pulpa o consistencia y su contenido de inorgánicos (como cenizas) de acuerdo a las Normas TAPPI T 240 om- 02 y T 211 om- 93 , respectivamente.

\section{Resultados y análisis}

\subsection{Materia prima}

La Tabla 3 presenta las características más importantes de la pulpa para cada uno de los grados de papel fabricados. En promedio, el contenido de inorgánicos como cenizas en la pulpa de pino es de $0.68 \%$, en la pulpa semiquímica un $1.14 \%$ y en la pulpa semiquímica del patio un $1.40 \%$.

Durante la producción de sacos, en donde la pulpa es más limpia, el contenido de inorgánicos como cenizas en promedio es de $1.10 \%$. Durante la producción de liner el porcentaje es de $1.62 \%$ y en la producción de corrugado medio es de $2.18 \%$, 
siendo este el valor más alto por su alto contenido de pulpa semiquímica.

\subsection{Sistema de limpieza}

Debido a la disposición de los hidrociclones, se encontró que la etapa primaria compromete la eficiencia total del sistema ya que recibe toda la pulpa y el aceptado va directo a la máquina de papel. Por esto se considera su eficiencia como la eficiencia global del sistema. En la etapa secundaria y terciaria solo clasifican los rechazos de la etapa primaria en un sistema de cascada y ayudan en la recuperación de fibra de la primera etapa.

La Tabla 4 presenta los resultados promedio de los muestreos. La mayor eficiencia del sistema se presenta en los muestreos realizados durante la producción de papel para liner en sus diferentes grados, obteniéndose un $24.1 \%$. Por otra parte, la eficiencia más baja se registró en la producción de corrugado medio con un $22.4 \%$.

Tabla 4. Consistencias y cenizas promedio en las entradas a la máquina y al espesador.

\begin{tabular}{lccc}
\hline \multicolumn{1}{c}{ Etapa Primaria } & Supersacks & $\begin{array}{c}\text { Corrugado } \\
\text { medio }\end{array}$ & Liner \\
\hline Cenizas Alimentación & 0.66 & 1.20 & 0.87 \\
Consistencia Alimentación & 0.70 & 1.02 & 0.80 \\
Cenizas Aceptados & 0.59 & 1.08 & 0.77 \\
Consistencia Aceptados & 0.79 & 1.02 & 0.87 \\
Eficiencia & 22.49 & 22.38 & 24.10 \\
\hline \multicolumn{1}{c}{ Etapa Secundaria } & & & \\
\hline Cenizas Alimentación & 0.64 & 1.17 & 0.78 \\
Consistencia Alimentación & 1.01 & 1.08 & 1.10 \\
Cenizas Aceptados & 0.56 & 1.10 & 0.73 \\
Consistencia Aceptados & 0.82 & 0.97 & 0.95 \\
Eficiencia & 29.51 & 25.30 & 26.05 \\
\hline \multicolumn{1}{c}{ Etapa Terciaria } & & & \\
\hline Cenizas Alimentación & 0.86 & 1.57 & 1.15 \\
Consistencia Alimentación & 1.08 & 1.09 & 1.16 \\
Cenizas Aceptados & 0.63 & 1.18 & 0.81 \\
Consistencia Aceptados & 0.87 & 0.92 & 1.00 \\
Cenizas Rechazo & 1.72 & 2.62 & 1.78 \\
Consistencia Rechazos & 1.86 & 1.25 & 1.36 \\
Eficiencia & 40.67 & 39.24 & 42.29 \\
\hline
\end{tabular}

La eficiencia de la etapa secundaria se encuentra entre 25.3 y $29.5 \%$, obteniéndose el mayor valor en la producción de sacos con una eficiencia de $29.5 \%$ y el menor valor en la producción de corrugado medio con $25.3 \%$.

En la etapa terciaria, la eficiencia se encuentra en el rango de 39.2 a $42.3 \%$, siendo el valor más alto de eficiencia en la producción de liner con $42.3 \%$ y el valor más bajo en la producción de corrugado medio con $39.2 \%$.

De acuerdo a los resultados, la etapa terciaria es la más eficiente (39.2-42.3\%) y la etapa primaria es la de menor eficiencia (22.4-24.1\%) para todos los grados de papel.

\subsubsection{Efecto del porcentaje de cenizas sobre el sistema de limpieza}

El mayor porcentaje de cenizas se presenta en corrugado medio con un $1.2 \%$ a la entrada al sistema de limpieza, a diferencia del porcentaje de cenizas en liner y sacos con un $0.9 \%$ y $0.7 \%$, respectivamente. Lo anterior, corresponde a las fórmulas que se utilizan para la elaboración de cada uno de los grados de papel, tomando como base que la pulpa semiquímica y de pino entran a la máquina de papel con un porcentaje de ceniza de $1.14 \%$ y $0.68 \%$, respectivamente. Además, la pulpa semiquímica que se encuentra en el patio antes de entrar al molino tiene un porcentaje de cenizas del $1.40 \%$, por estar en contacto con el medio ambiente.

Para los tres grados de papel, se aprecian muy pequeñas diferencias entre el porcentaje de cenizas de la entrada y aceptados de la etapa primaria y secundaria, a diferencia de la etapa terciaria, donde se observa una diferencia considerable en el porcentaje de cenizas.

\subsubsection{Efecto de la consistencia de entrada a la máquina sobre el sistema de limpieza}

En los muestreos realizados en corrugado medio, se encontraron los valores más altos de consistencias, con un intervalo de 0.94 a $1.15 \%$; encontrándose las eficiencias más altas a valores cercanos al $1.0 \%$ de consistencia. 
Durante la producción de sacos, los valores de consistencias en los muestreos se mantuvieron en un intervalo de 0.58 a $0.85 \%$, siendo éste el intervalo de consistencia más bajo utilizado en la producción de los tres grados de papel. Las eficiencias más altas para este grado de papel se encontraron a valores entre 0.65 y $0.75 \%$ de consistencia.

En los muestreos realizados durante la producción de liner, se obtuvieron valores de consistencias en un intervalo de 0.67 a $0.93 \%$ y con las eficiencias más altas a valores cercanos a $0.72 \%$ de consistencia.

\subsubsection{Efecto del nivel de aire en la suspensión de pulpa en el sistema de limpieza}

En general, la entrada y dispersión de aire en el sistema de la máquina de papel puede causar efectos negativos en la calidad y propiedades del papel. El valor de aire en el sistema no debe estar por encima de $0.4 \%$ (Sandoval \& Tovar, 2006).

Entre los problemas de producción que causa la entrada de aire y espuma en la máquina, se encuentran la disminución de la eficiencia de las bombas, la mala formación de la hoja, el menor drenaje en la mesa, la menor productividad, el crecimiento bacteriano, la flotación de suciedades, la variación en el gramaje del papel, la disminución de la tracción y la baja resistencia a la humedad.

El sistema de limpieza introduce aire ya que en algunos momentos la presión de salida en los hidrociclones en la corriente de aceptados es negativa y puede generar incorporación de aire a través de los aceptados. También, por ser un sistema abierto atmosférico, puede introducirse aire de la bandeja de rechazos, por desgaste de los conos o mala conexión de las mangueras, entre otras situaciones.

Por lo anterior, se realizaron mediciones del nivel de aire en la suspensión de pulpa, tomando muestras en la corriente de aceptados de la etapa primaria, a partir del equipo EGT (Entrained Gas Tester). Estos resultados se muestran en la Tabla 5.
Tabla 5. Medición de aire atrapado en la máquina de papeles Kraft.

\begin{tabular}{ccc}
\hline Material & $\begin{array}{c}\text { \% aire } \\
\text { atrapado }\end{array}$ & Observaciones \\
\hline Sacos & 1.10 & \\
Sacos & 1.54 & Alta espuma en la mesa \\
Sacos & 1.20 & Disminución espuma \\
Sacos & 1.10 & \\
Sacos & 1.34 & Espuma en la mesa \\
Sacos & 1.24 & \\
Sacos & 1.10 & \\
Liner & 1.09 & \\
Liner & 1.06 & \\
Liner & 1.11 & \\
Liner & 1.10 & Espuma en la mesa \\
Liner & 1.20 & \\
Corrugado Medio & 1.20 & Espuma en la mesa \\
Corrugado Medio & 1.10 & Alta espuma en la mesa \\
Corrugado Medio & 1.34 & \\
Corrugado medio & 1.56 &
\end{tabular}

No se encontró un cambio significativo a lo largo del periodo de muestreo y en todas las mediciones. En todo momento, el porcentaje de aire en la suspensión de pulpa se mantuvo alto, muy por encima del valor de $0.4 \%$ referenciado por proveedores lideres en el mercado mundial de antiespumantes y desespumantes. Según información técnica de dichos proveedores, los valores medidos son altos y afectan principalmente la formación del papel (Sandoval \& Tovar, 2006).

\subsection{Caracterización de contaminantes en el tanque sedimentador}

Se tomaron muestras en el tanque sedimentador para observar y clasificar las partículas contaminantes que provienen de la pulpa de alimentación a la máquina de papeles Kraft y de aquellas que permanecen en el sistema por efectos de recirculación.

Para la toma de muestras fue necesario drenar el tanque sedimentador con el fin de eliminar el exceso de agua y pulpa presentes en suspensión. Luego, para realizar la clasificación de las partículas, se eliminó la pulpa presente por métodos físicos y las partículas obtenidas se sometieron a un proceso de secado y tamizado de acuerdo a la norma ASTM E11. Los resultados del proceso de tamizado se muestran en la Tabla 6. 


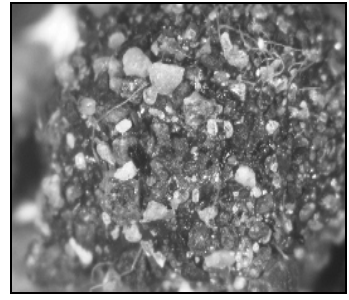

a.

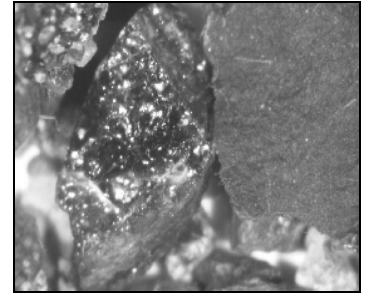

b.

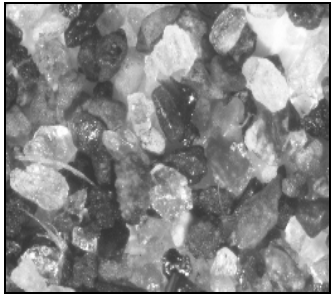

c.

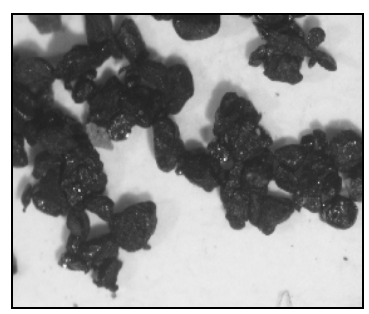

d.

Figura 4. Fotografías de contaminantes en la pulpa: a. aglomerado, b. resinato de Colofonia, c. arena y precipitado de alumbre, $d$. metales.

Tabla 6. Clasificación de partículas (Norma ASTM E11).

\begin{tabular}{ccrc}
\hline $\begin{array}{c}\text { Standard } \\
(\mathrm{mm})\end{array}$ & Malla alternativa & Peso (g) & Fracción (\%) \\
\hline 0.85 & 20 & 19.2040 & 20.3 \\
0.25 & 60 & 41.5979 & 43.9 \\
0.045 & 325 & 33.7208 & 35.6 \\
Colector & - & 0.1826 & 0.2 \\
Total & & 94.7053 & 100 \\
\hline
\end{tabular}

La mayor fracción (43.9\%) se encuentra retenida en la malla 60 que corresponde a un orificio de $0.25 \mathrm{~mm}$ de diámetro. Llama la atención la presencia de arena y resinatos de colofonia que muestran, la precipitación de los químicos empleados en el proceso. Mediante un microscopio estereoscópico a 63 aumentos, se observaron los contaminantes que se pueden apreciar en la Figura 4.

\section{Conclusiones}

La metodología empleada permitió una adecuada evaluación del sistema de limpieza.

El sistema de limpieza presenta una eficiencia de $23 \%$ en promedio, encontrándose, por debajo de las especificaciones de diseño.

La etapa determinante del proceso es la etapa primaria, debido a la configuración del sistema de hidrociclones.

Los hidrociclones son más eficientes durante la producción de papel liner, supersacks y corrugado medio, respectivamente, debido a las condiciones de operación en cada proceso (\% consistencia, fórmula, productividad).
El contenido de inorgánicos (cenizas) promedio en la suspensión de pulpa en los aceptados del sistema es de $0.59 \%$ para sacos, de $0.77 \%$ para liner y $1.08 \%$ para corrugado medio.

La consistencia de alimentación al sistema de limpieza debe mantenerse por debajo del $0.8 \%$, para un buen desempeño del equipo. Esto fue posible solamente en la producción de sacos con un intervalo de consistencia entre $0.58-0.85 \%$ y parcialmente, para liner con consistencias entre 0.67 y $0.93 \%$.

\section{Referencias bibliográficas}

Attwood, B.W., \& Baum, G.A. (1998). Pulp and paper manufacture. Toronto: McGraw Hill.

Merriman, K. (1993). Cleaning for contaminant removal in recycled-fiber systems. In: R.J. Spangenberg (editor), Secondary fiber recycling. Tappi Press, (Chapter 13).

Sandoval, L., \& Tovar, M. (2006). Evaluación de un sistema de limpieza para materiales fibrosos en una máquina de papeles Kraft. Trabajo de grado, Escuela de Ingeniería Química, Universidad del Valle, Cali, Colombia.

Sistema Bauer de Limpieza (1980). Reportes y manuales.

Smith, K. (1979). Federal's 100-tpd market pulp machine goes on-line at Riegelwood. Pulp and paper 21 (12), 20-25. 